\title{
Meta-analysis of the association of the haptoglobin genotype with cardiovascular outcomes and the pharmacogenomic interactions with vitamin E supplementation
}

This article was published in the following Dove Press journal: Pharmacogenomics and Personalized Medicine

\author{
Rabea Asleh ${ }^{1,2}$ \\ Alexandros Briasoulis ${ }^{3}$ \\ Elliot M Berinstein' \\ Joshua B Wiener' \\ Mohan Palla ${ }^{4}$ \\ Sudhir S Kushwaha ${ }^{2}$ \\ Andrew P Levy' \\ 'Bruce and Rappaport Faculty of \\ Medicine, Technion Institute of \\ Technology, Haifa, Israel; ${ }^{2}$ Department \\ of Cardiovascular Diseases, Mayo \\ Clinic, Rochester, MN, USA; ${ }^{3}$ Division \\ of Cardiovascular Diseases, University \\ of lowa Hospitals and Clinics, lowa \\ City, IA, USA; ${ }^{4}$ Department of \\ Cardiology, Detroit Medical Center, \\ Wayne State University, Detroit, MI, \\ USA
}

Objectives: The objectives of the study were to compile and summarize the data from all of the clinical trials designed to examine the association between haptoglobin (Hp) genotype and incidence of cardiovascular (CV) events in patients with diabetes mellitus (DM) and to assess the impact of vitamin $\mathrm{E}$ treatment on $\mathrm{CV}$ outcomes according to the Hp genotype.

Background: The Hp genotype could serve as a predictive biomarker to DM patients who may benefit from vitamin $\mathrm{E}$ therapy.

Methods: The electronic databases MEDLINE, PubMed, EMBASE and the Cochrane Library for Central Register of Clinical Trials were searched systematically using the following MESH terms: "haptoglobin genotype", "diabetes mellitus" and "cardiovascular events".

Results: Overall, 13 studies fit the inclusion criteria for this analysis, yielding a large study population that included 6,161 patients without Hp 2-2 and 4,684 patients with Hp 2-2. The analysis of these studies showed that the incidence of CV events in DM patients with the Hp 2-2 genotype was significantly increased as compared to non-Hp 2-2 patients in all three subgroups of case-control (OR: 2.2, 95\% CI: 1.3-3.6; $P=0.003$ ), cohort (OR: 1.3, 95\% CI: $1.2-1.5 ; P=0.001$ ) and randomized controlled trials (OR: 1.6, 1.2-2.2; $P=0.005$ ). Among patients with the Hp 2-2 genotype, administration of vitamin $\mathrm{E}$ was associated with lower rates of $\mathrm{CV}$ events (OR: 0.66 , 95\% CI: $0.45-0.95 ; P=0.025)$. Further investigation into the association between Hp 2-2 and myocardial infarction, stroke, mortality and end-stage renal disease was also performed.

Conclusion: The Hp genotype is a risk factor for CV events in patients with DM, and administration of vitamin E appears to offer a low cost and accessible means of reducing CV events and mortality in this population.

Keywords: haptoglobin genotype, diabetes mellitus, vitamin E, cardiovascular disease, antioxidants, HDL dysfunction

\section{Introduction}

Cardiovascular disease (CVD) is the leading cause of morbidity and mortality in patients with diabetes mellitus (DM). Due to the enormous cardiovascular $(\mathrm{CV})$ burden associated with DM, reduction in $\mathrm{CV}$ risk is one of the main goals in the treatment of DM. In 2012, the cost of the treatment of DM was estimated to be $>245$ billion dollars a year in the USA alone, representing the biggest economic burden to the US health-care system, with a large portion spent on the treatment of CV complications.

The increased risk of CVD in patients with DM is partially due to increased oxidative stress mediated by the generation of reactive oxygen species in hyperglycemic
Bruce and Rappaport Faculty of Medicine, Technion Institute of Technology, I Efron Street, Bat Galim, Haifa 3525433, Israel

$\mathrm{Tel}+97248295202$

Fax +972485I 4I03

Email alevy@tx.technion.ac.il 
states leading to oxidative modifications of low-density lipoprotein (LDL), high-density lipoprotein (HDL) and various proteins within the vessel wall, thus increasing their thermogenic potential. ${ }^{2,3}$ One of the major consequences of these oxidative modifications is that HDL can lose its protective anti-inflammatory properties as well as the ability to shuttle cholesterol back to the liver through reverse cholesterol transport.

Beyond the traditional risk factors for CVD, genetic susceptibility is a major determinant of CV risk, including patients with DM. One such genetic biomarker is the haplotype for the gene that encodes the haptoglobin ( $\mathrm{Hp}$ ) protein at the chromosome $16 \mathrm{q} 22$ locus. ${ }^{4} \mathrm{Hp}$ is an $\alpha 2$-sialoglycoprotein synthesized by hepatocytes to protect blood vessels and the kidneys from adverse oxidative effects and tissue injury induced by extracorpuscular free hemoglobin $(\mathrm{Hb})$ released during intravascular lysis of erythrocytes amounting to several grams of free $\mathrm{Hb}$ each day. ${ }^{5-7}$ There are two major common classes of alleles for this gene, Hp 1 and $\mathrm{Hp} 2$, with the Hp 2 allele probably arising from a duplication event of exons 3 and 4 of the Hp 1 allele early in human evolution. ${ }^{4}$ In the Western world, in both healthy and patients with DM, the genotype frequency of $\mathrm{Hp} \mathrm{1-1,} \mathrm{Hp} \mathrm{1-2} \mathrm{and} \mathrm{Hp} \mathrm{2-2} \mathrm{is} 16 \%$, $48 \%$ and $36 \%$, respectively. ${ }^{8,9}$ Previous studies have shown that $\mathrm{Hp}$ 2-2 protein is an inferior antioxidant compared with other Hp proteins with a resultant increase in Hb-driven lipoprotein oxidative modifications, HDL dysfunction, increased macrophage inflammation and apoptosis, thus leading to increased atherogenicity, specifically in the setting of DM. ${ }^{10-15}$ The increased oxidative stress seen in both in vitro and in vivo patients with DM and the Hp 2-2 genotype suggests antioxidants as a potential target for mitigating CVD risk among this group. Vitamin E administration is a potential treatment that could provide an affordable, accessible and effective benefit to patients with high oxidative stresses on their CV system. Vitamin $E$ is a group of eight lipophilic molecules, $\alpha-, \beta$-, $\gamma$ - and $\delta$-tocopherol and $\alpha$-, $\beta$-, $\gamma$ - and $\delta$-tocotrienol, which acts as antioxidants by scavenging free radicals and singlet oxygen. ${ }^{16,17}$ Despite encouraging preclinical data, early clinical trials failed to show a benefit of vitamin E supplementation on preventing CVD. ${ }^{18}$ It is possible that this was due to a failure to identify specific patient populations with increased oxidative stress that would benefit from vitamin E therapy.

Multiple clinical studies have investigated the effect of Hp genotype on CV clinical outcomes with most, but not all, showing an increased incidence of CV events and CV death among patients with DM and the Hp 2-2 genotype. This study has two main aims. First, we sought to compile and summarize the data from all of the published clinical trials related to the incidence of $\mathrm{CV}$ events in patients with $\mathrm{DM}$, according to their Hp genotype. This includes data from 13 trials that looked at multiple outcomes, including myocardial infarction (MI), stroke, CV death and end-stage renal disease (ESRD). Second, we sought to compile and assess all of the data on the clinical benefit of vitamin E treatment among patients with DM based on their Hp genotype.

\section{Methods}

\section{Search strategy}

The electronic databases MEDLINE, PubMed, Embase and the Cochrane Library for Central Register of Clinical Trials were searched systematically using the following MESH terms: "haptoglobin 2-2 genotype", "diabetes mellitus" and "cardiovascular events". The search was limited to the studies in human subjects and peer-reviewed journals in English language published until February 2017. The two reviewers identified a total of 530 studies, after the independent electronic search. There were no disagreements in the literature search between the reviewers. Subsequently, the references listed in the 13 studies identified by the electronic search were manually searched. Manual search did not reveal any new studies apart from the ones identified by the electronic search.

\section{Study selection}

All observational studies published as original articles in peer-reviewed scientific journals in English were included. Studies were selected based on inclusion criteria: patient population: group of patients with DM (type 1 or type 2) in accordance with the standard clinical guidelines, use of Hp genotyping or phenotyping and comparison of outcomes between patients with and without the Hp 2-2 genotype. In the subgroup analysis of prospective randomized controlled trials (RCTs), vitamin E was administered in patients with type $2 \mathrm{DM}$ and the Hp 2-2 genotype. Those trials that did not report any of the following variables or outcomes: number of events in both the intervention and reference groups, length of study, description of the main relevant features of the study population, including gender and age, were excluded. The eligibility was not restricted according to the outcomes.

\section{Data extraction and quality}

The data were independently extracted by two authors (R.A. and A.B.) using the standardized protocol and reporting form. Disagreements were resolved by open discussion, and consensus was reached after discussion. 
The study characteristics such as study design, sample size, baseline demographics and CV risk factors, inclusion criteria and exclusion criteria, primary and secondary outcomes and follow-up duration and demographic characteristics of the patients were extracted.

\section{Outcome assessment}

The primary outcome measure was incident CV events, including fatal and nonfatal MI, stroke, heart failure and need for revascularization. Death or all-cause mortality, MI, CV death, stroke and ESRD were also examined. Four studies included MI (Heart Outcomes Prevention Evaluation [HOPE] double placebo, Israeli Cardiovascular Vitamin E [ICARE], Women's Health Study [WHS] and MUNICH post-PCA), two studies included all-cause mortality (Diabetes Heart Study [DHS] and WHS), two studies included ESRD (Diabetes Control and Complications Trial [DCCT] and Epidemiology of Diabetes Complications [EDC]), three studies included stroke (HOPE double placebo, ICARE and WHS) and five studies included CV death (HOPE double placebo, ICARE, WHS, DHS and MUNICH post-PCA) as part of their outcomes.

\section{Risk of bias}

The Cochrane's risk of bias tool was utilized in order to assess the individual risk of bias of each RCT. The Newcastle-Ottawa tool was used for the quality assessment of cohort studies. Two authors independently assessed the risk of bias and quality of studies in each eligible trial. This scale appoints a maximum of nine stars to each study: four stars for the adequate selection of the two groups, two stars for comparability of groups on the basis of the design and analysis and three stars for the adequate ascertainment of the exposure in both groups. High-quality studies received nine stars and medium-quality studies seven or eight stars.

\section{Data analysis and synthesis}

An intention-to-treat traditional meta-analysis was performed in line with recommendations from the Cochrane Collaboration and the Preferred Reporting Items for Systematic Reviews and Meta-Analyses Statement. All analyses were performed by Review Manager (RevMan) 5.3 (Copenhagen: The Nordic Cochrane Centre, The Cochrane Collaboration, 2011). The $\chi^{2}$ test of heterogeneity and the $I^{2}$ statistic of inconsistency were used to assess heterogeneity between studies. Statistically significant heterogeneity was defined as a $\chi^{2} P$-value $<0.05$ or an $I^{2}$ statistic $>75 \%$. In the absence of heterogeneity, pooled estimates of relative risks with their $95 \%$ CIs were calculated using the Mantel-Haenszel method. A DerSimonian-Laird random effects model for OR estimation for all outcomes was used in the presence of heterogeneity. Reported values are two-tailed, and hypothesis testing results were considered statistically significant when $P<0.05$. Small study effect, including publication bias, was tested using the funnel plot and the Egger's test. If publication bias was found, the nonparametric trim and fill method of Duval and Tweedie was performed to add studies that appeared to be missing.

\section{Results \\ Study selection}

Our search strategy is outlined in Figure 1. Literature search resulted in a total of 13 studies including three prospective RCTs, three case-control and seven cohort studies, which were analyzed separately for each outcome. All of them were reported between 2000 and 2016. For the end point of CV events, subgroup analysis was performed for RCTs, cohort and case-control studies. Additionally, the effects of vitamin $\mathrm{E}$ on $\mathrm{CV}$ events in patients with and without the $\mathrm{Hp}$ 2-2 genotype were analyzed.

\section{Study characteristics}

The total number of patients without Hp 2-2 was 6,161 and with Hp 2-2 was 4,684. The baseline characteristics of the included studies are represented in Table 1. Among Hp 2-2 patients included in the analysis of the three RCTs, 1,094 were randomized to receive vitamin E supplementation and 1,016 to receive placebo.

\section{Quality assessment}

On the basis of quality assessment, all studies were deemed to be high quality. No low-quality studies were included.

\section{CV events}

Eleven studies reported data on CV events (CVD events). The incidence of CVD events in DM patients with the Hp 2-2 genotype was significantly increased as compared to patients without the Hp 2-2 genotype in all three subgroups of case-control studies (OR: 2.18, 95\% CI: 1.31-3.61, $P=0.003$; Figure 2A), in cohort studies (OR: $1.31,95 \% \mathrm{CI}$ : $1.12-1.55 ; P=0.001$; Figure $2 \mathrm{~A}$ ) and in RCTs (OR: $1.60,95 \%$ CI: $1.15-2.22 ; P=0.005$; Figure $2 \mathrm{~A})$, without significant heterogeneity between trials $\left(I^{2}=0 \%, P=0.37 ; I^{2}=46.7 \%, P=0.38\right.$; and $P=26.36 \%, P=0.26$, respectively). Among patients with the Hp 2-2 genotype, administration of vitamin $E$ was associated with lower rates of CVD events (OR: $0.66,95 \%$ CI: 


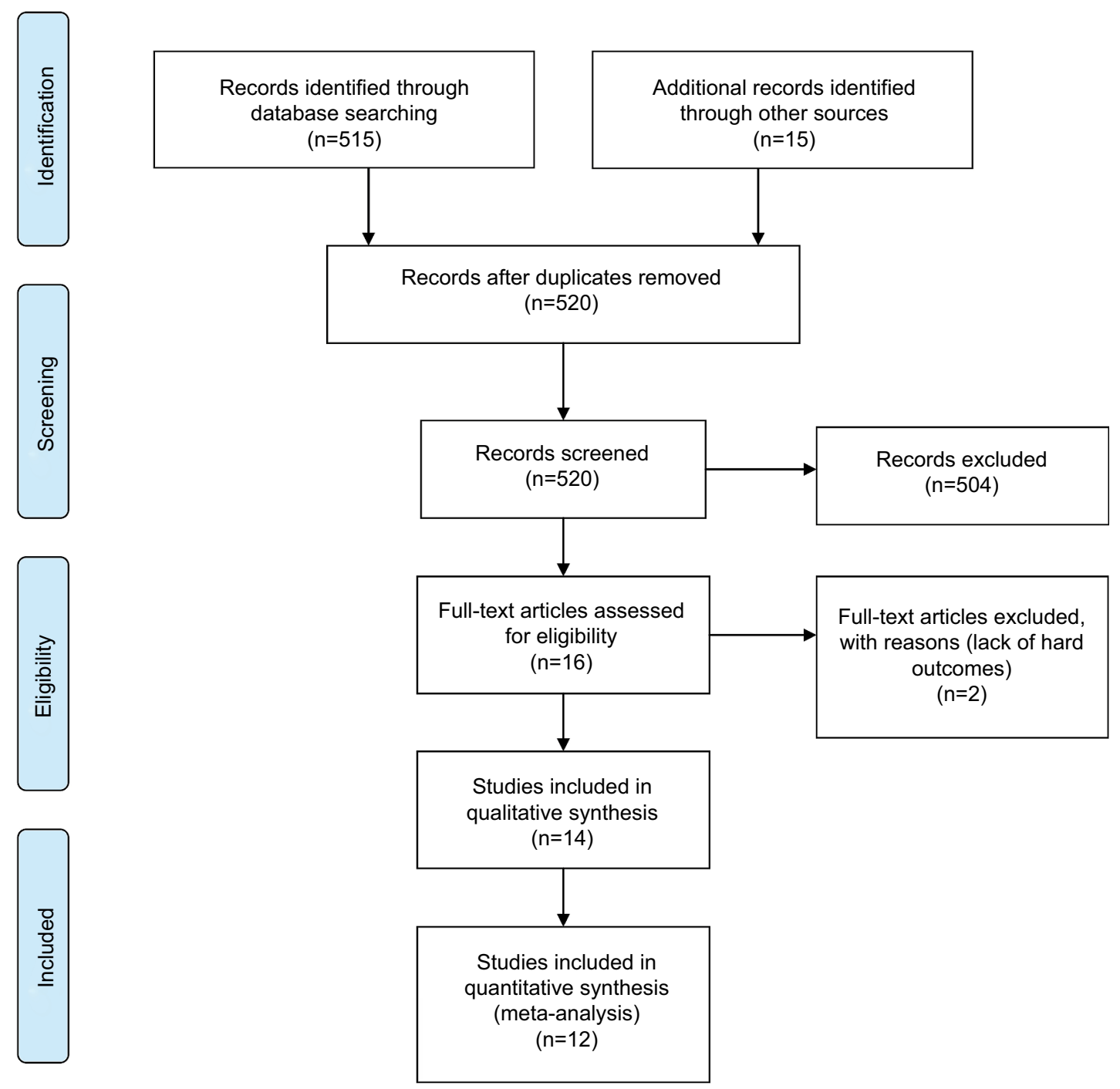

Figure I Search strategy for studies used in our meta-analysis.

$0.45-0.95 ; P=0.025$; Figure $2 \mathrm{~B})$, without significant heterogeneity between trials $\left(I^{2}=16.16 \%, P=0.30\right)$. Among non-Hp 2-2 patients, administration of vitamin E did not significantly affect the rates of CVD events compared with placebo (OR: 1.11, 95\% CI: 0.80-1.53, $P=0.50$; Figure 2C), without significant heterogeneity between trials $\left(I^{2}=0 \%, P=0.45\right)$.

\section{Myocardial infarction}

Five studies reported data on the incidence of MI: three RCTs (ICARE, WHS and HOPE) and two cohort studies (DCCT/ Epidemiology of Diabetes Interventions and Complications and MUNICH post-PCA). MI rates were significantly increased in patients with Hp 2-2 in both the RCT subgroup (OR: 1.45, 95\% CI: 1.01-2.07, $P=0.043$; Figure 3A) and cohort studies (OR: 1.86, 95\% CI: 1.19-2.90, $P=0.006$; Figure 3A) without significant heterogeneity between tri- als $\left(I^{2}=0 \%, P=0.53\right.$ and $I^{2}=36.68 \%, P=0.21$, respectively). Vitamin E administration was associated with numerically lower but not statistically significant rates of $\mathrm{MI}$ in patients with the Hp 2-2 genotype (OR: 0.62, 95\% CI: 0.35-1.11, $P=0.10 ; I^{2}=39.34 \%, P=0.19$; Figure $\left.3 \mathrm{~B}\right)$.

\section{Mortality}

Two studies reported data on the incidence of all-cause mortality (DHS and WHS) and four studies on CV mortality (DHS, WHS, ICARE and HOPE). The presence of Hp 2-2 genotype was not associated with higher all-cause mortality (OR: 1.19, 95\% CI: 0.93-1.52; $P=0.17$; Figure 4). CV death rates were not significantly different among patients with the Hp 2-2 genotype compared with patients carrying other genotypes in the RCT subgroup (OR: 2.17, 95\% CI: 0.71-6.68; $P=0.18$; Figure 5A), with significant heterogeneity between 


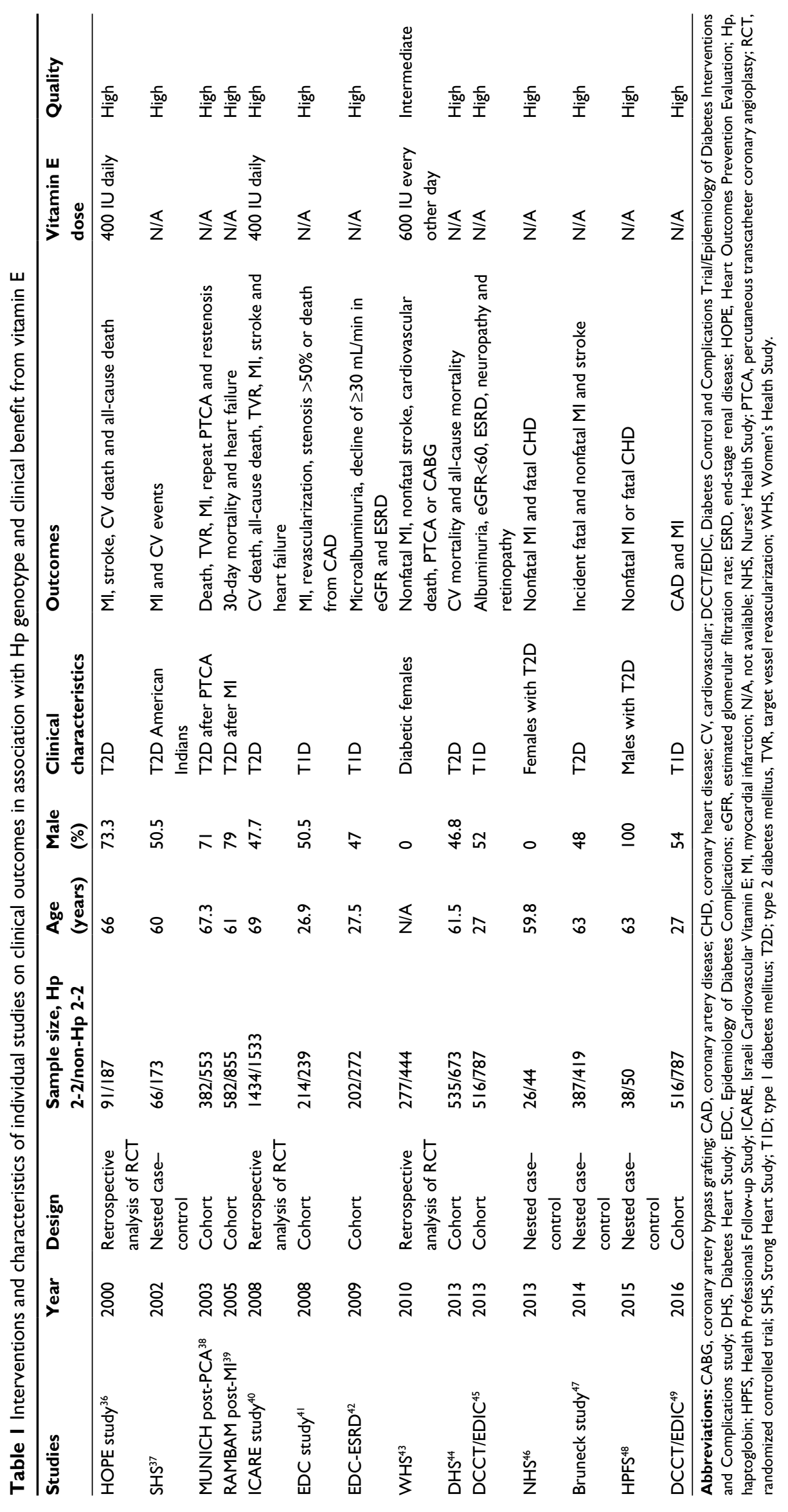


A

\begin{tabular}{|c|c|c|c|}
\hline Studies & Estimate $(95 \% \mathrm{Cl})$ & Ev/HP 2-2 & Ev/non-HP 2 \\
\hline $\mathrm{SHS}^{37}$ & $2.507(1.325-4.743)$ & $50 / 66$ & $96 / 173$ \\
\hline $\mathrm{NHS}^{46}$ & $4.000(0.811-19.728)$ & $24 / 26$ & $33 / 44$ \\
\hline HPFS $^{48}$ & $1.253(0.475-3.305)$ & $29 / 38$ & $36 / 50$ \\
\hline Subgroup case-control studies $\left(I^{2}=0 \%, P=0.369\right)$ & $2.176(1.313-3.607)$ & $103 / 130$ & $165 / 267$ \\
\hline MUNICH post-PCA 38 & $1.364(1.022-1.821)$ & $120 / 382$ & $139 / 553$ \\
\hline EDC study ${ }^{41}$ & $1.542(1.029-2.312)$ & $74 / 214$ & $61 / 239$ \\
\hline Bruneck $^{47}$ & $0.960(0.654-1.410)$ & $58 / 387$ & $65 / 419$ \\
\hline DCCT/EDIC ${ }^{45}$ & $1.244(0.882-1.755)$ & $66 / 616$ & $83 / 787$ \\
\hline $\mathrm{DHS}^{44}$ & $1.599(1.060-2.413)$ & $55 / 535$ & $45 / 673$ \\
\hline Subgroup cohort studies $\left(l^{2}=46.7 \%, P=0.380\right)$ & $1.314(1.115-1.548)$ & $373 / 2,034$ & $393 / 2,671$ \\
\hline HOPE study ${ }^{36}$ & $2.183(1.223-3.896)$ & $29 / 91$ & $33 / 187$ \\
\hline ICARE study ${ }^{40}$ & $1.714(1.087-2.704)$ & $49 / 1,434$ & $31 / 1,533$ \\
\hline $\mathrm{WHS}^{43}$ & $1.209(0.776-1.884)$ & $39 / 277$ & $53 / 444$ \\
\hline Subgroup RCT $\left(I^{2}=26.36 \%, P=0.257\right)$ & $1.597(1.151-2.215)$ & $117 / 1,802$ & $117 / 2,164$ \\
\hline Overall $\left(I^{2}=23.71 \%, P=0.218\right)$ & $1.444(1.231-1.694)$ & $593 / 3,966$ & $675 / 5,102$ \\
\hline
\end{tabular}

B

Studies

HOPE study ${ }^{36}$

ICARE study ${ }^{40}$

WHS $^{43}$

Overall $\left(I^{2}=16.16 \%, P=0.303\right)$

C

Studies

HOPE study ${ }^{36}$

WHS $^{43}$

Overall $\left(I^{2}=0 \%, P=0.450\right)$
$0.656(0.454-0.948)$

\section{Estimate $(95 \% \mathrm{Cl})$}

$0.685(0.416-1.128)$

$0.461(0.251-0.845)$

$0.936(1.475-1.842)$

$73 / 1,094$

Ev/Vitamin E

$60 / 326$

$29 / 215$

$89 / 541$

$1.332(0.749-2.369)$

$1.108(0.802-1.530)$
Ev/Vitamin E

$37 / 222$

$16 / 726$

20/146

$33 / 708$

$19 / 131$

$92 / 1,016$

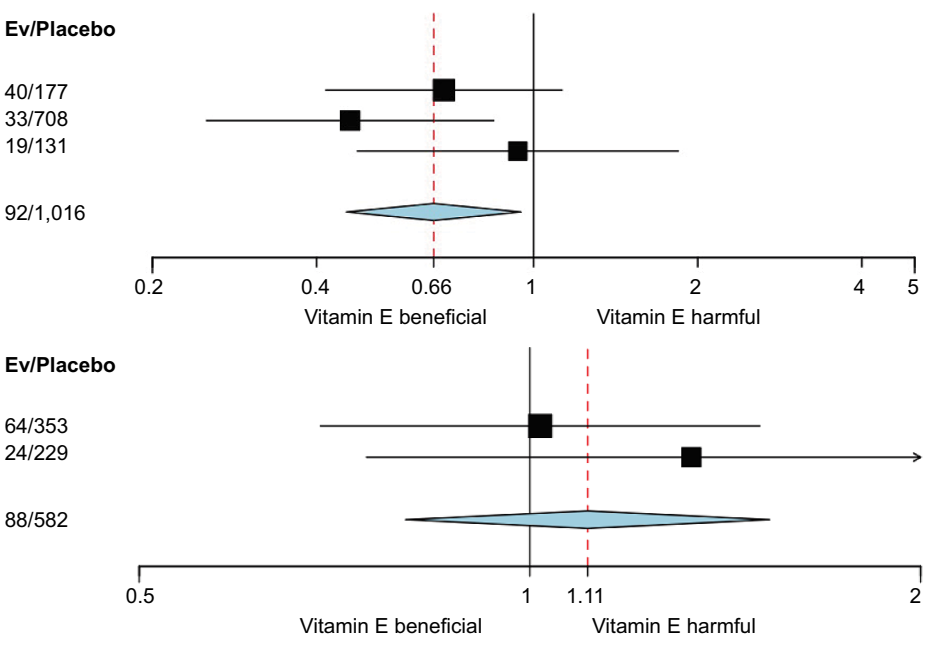

Ev/Placebo

Vitamin E beneficial

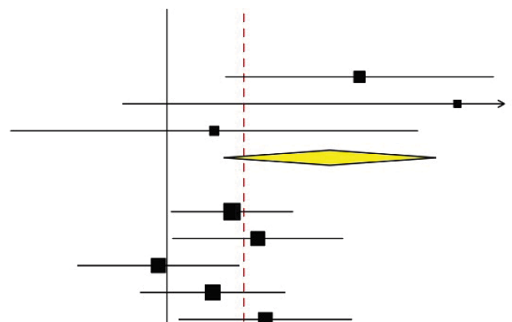

$<$
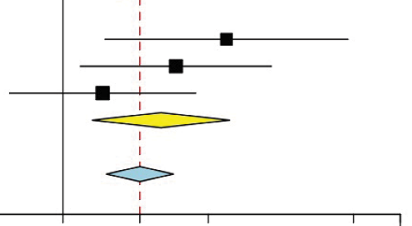

1.442

西

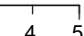

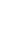

Figure $2 \mathrm{Hp}$ 2-2 genotype and risk of CV events and effect of vitamin E on major CV outcomes.

Notes: (A) Random effect meta-analysis of cohort studies and RCTs for CV events between Hp 2-2 and non-Hp 2-2 genotypes. The figure presents number of events, number of patients in treatment and control groups, OR and $95 \% \mathrm{Cl}$ for each trial, overall OR estimate with $95 \% \mathrm{Cl}$ and $P$ value for association test, $P$ value for heterogeneity test and between trial inconsistency $\left(I^{2}\right)$ measures. (B) Random effect meta-analysis of the effects of vitamin $\mathrm{E}$ administration on CV events in diabetic patients with the $\mathrm{HP}$ 2-2 genotype. (C) Random effect meta-analysis of the effects of vitamin E administration on CV events in diabetic patients without the Hp 2-2 genotype.

Abbreviations: CV, cardiovascular; DCCT/EDIC, Diabetes Control and Complications Trial/Epidemiology of Diabetes Interventions and Complications Study; DHS, Diabetes Heart Study; EDC, Epidemiology of Diabetes Complications; HOPE, Heart Outcomes Prevention Evaluation; Hp, haptoglobin; HPFS, Health Professionals Followup Study; ICARE, Israeli Cardiovascular Vitamin E; NHS, Nurses' Health Study; RCT, randomized controlled trial; SHS, Strong Heart Study; WHS, Women's Health Study.

studies $\left(I^{2}=68.43 \%, P=0.042\right)$. However, supplementation of vitamin $\mathrm{E}$ resulted in significantly lower rates of $\mathrm{CV}$ death among patients with Hp 2-2 (OR: 0.47, 95\% CI: 0.26-0.85; $P=0.013$; Figure 5B), without significant heterogeneity between trials $\left(I^{2}=0 \%, P=0.93\right)$.

\section{Stroke}

Three RCTs reported data on the incidence of stroke (WHS, ICARE and HOPE). The presence of Hp 2-2 genotype was not associated with higher incidence of stroke (OR: 1.48, 95\% CI: $0.95-2.30 ; P=0.085$; Figure 6A), without significant heterogeneity between trials $\left(I^{2}=0 \%, P=0.47\right)$. Supplementation of vitamin $\mathrm{E}$ did not significantly decrease rates of stroke among patients with the Hp 2-2 genotype (OR: 0.78,
95\% CI: 0.44-1.37; $P=0.39$; Figure 6B), without significant heterogeneity between trials $\left(I^{2}=0 \%, P=0.47\right)$.

\section{End-stage renal disease}

Two studies reported data on the incidence of ESRD (DCCT and Epidemiology of Diabetes Complications). The ESRD rates were significantly increased in patients with the Hp 2-2 genotype (OR: 1.66, 95\% CI: 1.04-2.64; $P=0.03$; Figure 7), without significant heterogeneity between trials $(I=0 \%, P=0.56)$.

\section{Publication bias}

The funnel plot did not show asymmetry consistent with publication bias, and the Egger's test was not significant for the outcomes studied. 
A

\begin{tabular}{|c|c|c|c|}
\hline Studies & Estimate $(95 \% \mathrm{Cl})$ & Ev/HP 2-2 & Ev/non-HP 2-2 \\
\hline HOPE study ${ }^{36}$ & $1.944(1.021-3.703)$ & $21 / 91$ & $25 / 187$ \\
\hline ICARE study ${ }^{40}$ & $1.356(0.740-2.487)$ & $24 / 1,434$ & $19 / 1,533$ \\
\hline WHS $^{43}$ & $1.184(0.642-2.182)$ & $19 / 277$ & $26 / 444$ \\
\hline Subgroup RCT $\left(l^{2}=0 \%, P=0.532\right)$ & $1.447(1.011-2.069)$ & $64 / 1,802$ & $70 / 2,164$ \\
\hline MUNICH post-PCA ${ }^{38}$ & $2.437(1.371-4.329)$ & $32 / 382$ & $20 / 553$ \\
\hline DCCT/EDIC ${ }^{49}$ & $1.537(0.999-2.366)$ & $44 / 516$ & $45 / 787$ \\
\hline Subgroup cohort studies $\left(I^{2}=36.68 \%, P=0.209\right)$ & $1.858(1.191-2.897)$ & $76 / 898$ & $65 / 1,340$ \\
\hline Overall $\left(I^{2}=0 \%, P=0.457\right)$ & $1.444(1.231-1.694)$ & $593 / 3,966$ & $675 / 5,102$ \\
\hline
\end{tabular}

$\begin{array}{llll} & & & \\ \text { B } & & & \\ \text { Studies } & \text { Estimate }(95 \% \mathrm{Cl}) & \text { Ev/Vitamin E } & \text { Ev/Placebo } \\ & & & \\ \text { HOPE study } & & \\ \text { ICARE study } & & & \\ \text { WHS } & & & \\ & 0.544(0.305-0.972) & 23 / 222 & 17177 \\ & 0.396(0.163-0.960) & 7 / 726 & 17 / 708 \\ \text { Overall }\left(I^{2}=39.34 \%, P=0.192\right) & 0.618(0.346-1.106) & 41 / 1,094 & \\ & 1.253(0.488-3.216) & 11 / 146 & \\ & & \end{array}$
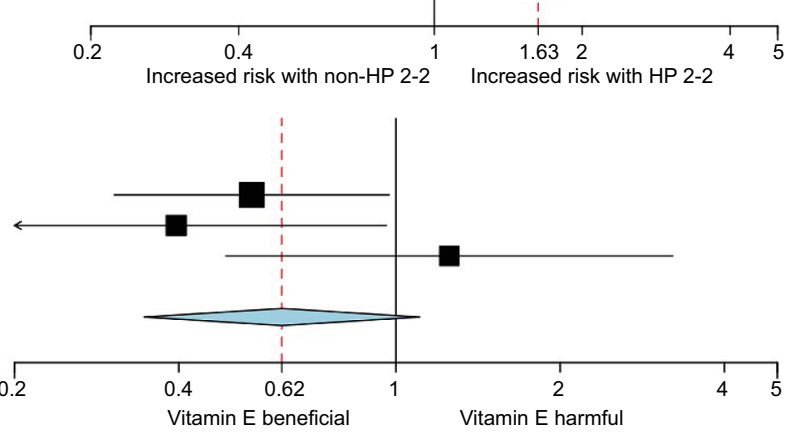

Figure $3 \mathrm{Hp}$ 2-2 genotype and risk of $\mathrm{Ml}$ and effect of vitamin $\mathrm{E}$ on $\mathrm{Ml}$ risk.

Notes: (A) Random effect meta-analysis of cohort studies and RCTs for Ml between Hp 2-2 and non-Hp 2-2 genotypes. (B) Random effect meta-analysis of the effects of vitamin $\mathrm{E}$ administration on $\mathrm{Ml}$ in diabetic patients with the $\mathrm{Hp}$ 2-2 genotype.

Abbreviations: DCCT/EDIC, Diabetes Control and Complications Trial/Epidemiology of Diabetes Interventions and Complications Study; HOPE, Heart Outcomes Prevention Evaluation; Hp, haptoglobin; ICARE, Israeli Cardiovascular Vitamin E; MI, myocardial infarction; RCT, randomized controlled trial; WHS, Women's Health Study.

$\begin{array}{lccc}\text { Studies } & \text { Estimate }(\mathbf{9 5} \% \mathbf{C l}) & \text { Ev/HP 2-2 } & \text { Ev/non-HP 2-2 } \\ & & & \\ \text { DHS } & & \\ \text { Subgroup cohort studies }\left(I^{2}=\mathrm{NA}, P=\mathrm{NA}\right) & 1.268(0.947-1.698) & 109 / 535 & 113 / 673 \\ & 1.268(0.947-1.698) & 109 / 535 & 113 / 673 \\ \text { WHS }^{43} & & & \\ \text { Subgroup RCT }\left(I^{2}=\mathrm{NA}, P=\mathrm{NA}\right) & 1.014(0.649-1.586) & 36 / 277 & 57 / 444 \\ & 1.014(0.649-1.586) & 36 / 277 & 57 / 444 \\ \text { Overall }\left(I^{2}=0 \%, P=0.412\right) & 1.186(0.929-1.515) & 145 / 812 & 170 / 1,117\end{array}$

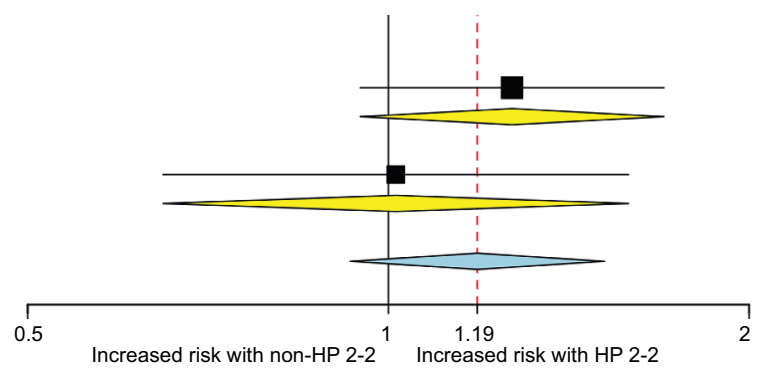

Figure $4 \mathrm{Hp}$ 2-2 genotype and risk of all-cause mortality.

Note: Random effect meta-analysis of cohort studies and RCTs for all-cause mortality between Hp 2-2 and non-Hp 2-2 genotypes.

Abbreviations: DHS, Diabetes Heart Study; Hp, haptoglobin; RCT, randomized controlled trial; WHS, Women's Health Study.

\section{Discussion}

Despite the importance of the common Hp copy number variant polymorphism, which determines Hp structure and affects its function, the relationship of copy number variant with genome-wide association is challenging. ${ }^{19}$ The reason for this complex association is that there is no strong linkage disequilibrium with any individual single nucleotide polymorphism (SNP) to allow one to successfully determine the Hp genotype with array-based copy number analysis or low-coverage sequencing. ${ }^{19-21}$ Therefore, Hp typing for each individual remains possible only with serum protein electrophoresis ${ }^{22}$ or polymerase chain reaction, ${ }^{23,24}$ which restricts the size and power of clinical studies to demonstrate association with clinical outcomes. In this systematic review and meta-analysis, the results of relevant association stud- ies in the literature were pooled to provide strong evidence that the Hp 2-2 genotype is associated with increased risk of CV disease among patients with DM as compared to those with other Hp genotypes. The results of the RCTs also demonstrate that vitamin $\mathrm{E}$ administration provides significant clinical benefit to DM patients with the Hp 2-2 genotype. Although there was no significant difference in CV mortality between Hp patient groups, vitamin E administration resulted in a significant decrease in $\mathrm{CV}$ mortality and the composite of CV events in the Hp 2-2 DM cohort, whereas no such benefit was seen with vitamin $\mathrm{E}$ therapy in the non-Hp 2-2 cohort. These results illustrate the potential benefit of selective vitamin $\mathrm{E}$ administration for the reduction of CV adverse clinical outcomes among DM patients with the Hp 2-2 genotype. 
A

$\begin{array}{llll}\text { Studies } & \text { Estimate }(\mathbf{9 5} \% \mathbf{C l}) & \text { Ev/HP 2-2 } & \text { Ev/non-HP 2-2 } \\ & & & \\ \text { DHS } & & \\ \text { Subgroup cohort studies }\left(I^{2}=\mathrm{NA}, P=\mathrm{NA}\right) & 1.599(1.060-2.413) & 55 / 535 & 45 / 673 \\ & 1.599(1.060-2.413) & 55 / 535 & 45 / 673 \\ & & & \\ \text { HOPE study } & & & \\ \text { ICARE study } & & & \\ \text { WHS } & 2.855(1.309-6.231) & 16 / 91 & 13 / 187 \\ \text { Subgroup RCT }\left(I^{2}=68.43 \%, P=0.042\right) & 8.595(1.074-68.803) & 8 / 1,434 & 1 / 1,533 \\ & 0.851(0.356-2.033) & 8 / 277 & 15 / 444 \\ \text { Overall }\left(I^{2}=54.11 \%, P=0.088\right) & 2.171(0.705-6.684) & 32 / 1,802 & 29 / 2,164 \\ & & & \\ & 1.814(0.987-3.333) & 87 / 2,337 & 74 / 2,837\end{array}$

B

Studies

HOPE study ${ }^{36}$

ICARE study ${ }^{40}$

WHS $^{43}$

Overall $\left(I^{2}=0 \%, P=0.929\right)$

$\begin{array}{ll}13 / 222 & 22 / 177 \\ 3 / 726 & 5 / 708\end{array}$

$3 / 146$

19/1,094

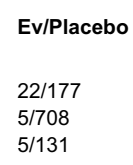

$32 / 1,016$
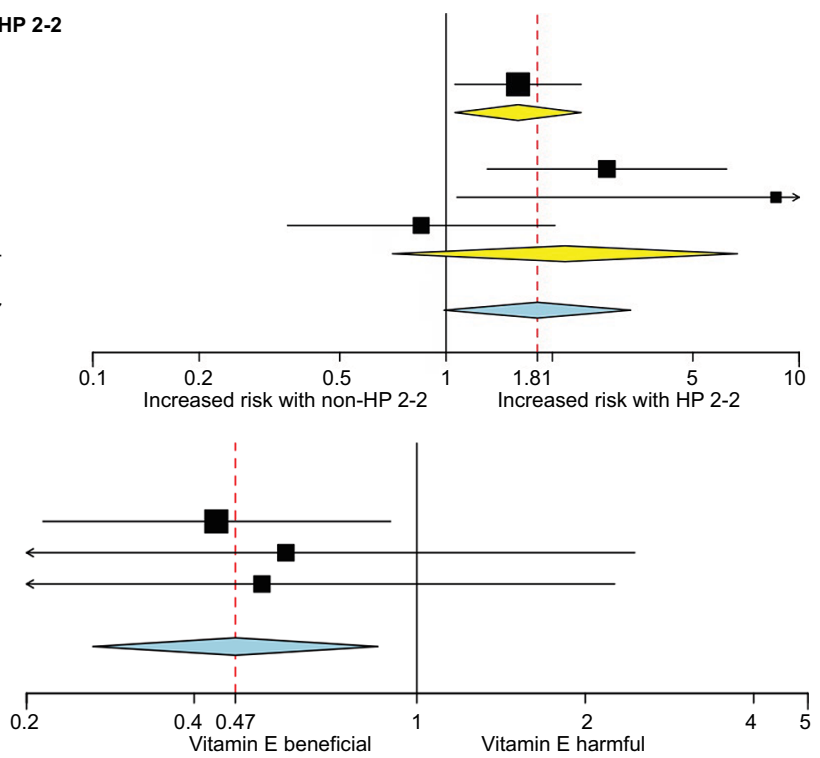

Figure 5 Hp 2-2 genotype and risk of CV mortality and the effect of vitamin E on CV mortality risk.

Notes: (A) Random effect meta-analysis of cohort studies and RCTs for CV mortality between Hp 2-2 and non-Hp 2-2 genotypes. (B) Random effect meta-analysis of the effects of vitamin $\mathrm{E}$ administration on $\mathrm{CV}$ mortality in diabetic patients with the $\mathrm{Hp}$ 2-2 genotype.

Abbreviations: CV, cardiovascular; DHS, Diabetes Heart Study; HOPE, Heart Outcomes Prevention Evaluation; Hp, haptoglobin; ICARE, Israeli Cardiovascular Vitamin E; RCT, randomized controlled trial; WHS, Women's Health Study.

\begin{tabular}{|c|c|c|c|}
\hline $\begin{array}{l}\text { A } \\
\text { Studies }\end{array}$ & Estimate $(95 \% \mathrm{Cl})$ & Ev/Vitamin E & Ev/Placebo \\
\hline HOPE study ${ }^{36}$ & $2.478(0.870-7.063)$ & $8 / 91$ & $7 / 187$ \\
\hline ICARE study ${ }^{40}$ & $1.137(0.573-2.260)$ & $17 / 1,434$ & $16 / 1,533$ \\
\hline WHS 43 & $1.540(0.765-3.100)$ & $16 / 277$ & $17 / 444$ \\
\hline Overall $\left(I^{2}=0 \%, P=0.470\right)$ & $1.478(0.948-2.304)$ & $41 / 1,802$ & $40 / 2,164$ \\
\hline \multicolumn{4}{|l|}{ B } \\
\hline Studies & Estimate $(95 \% \mathrm{Cl})$ & Ev/Vitamin E & Ev/Placebo \\
\hline HOPE study ${ }^{36}$ & $1.207(0.482-3.021)$ & $12 / 222$ & $8 / 177$ \\
\hline ICARE study ${ }^{40}$ & $0.528(0.194-1.436)$ & $6 / 726$ & $11 / 708$ \\
\hline $\mathrm{WHS}^{43}$ & $0.683(0.247-1.888)$ & $7 / 146$ & $9 / 131$ \\
\hline Overall $\left(I^{2}=0 \%, P=0.467\right)$ & $0.780(0.444-1.370)$ & $25 / 1,094$ & $28 / 1,016$ \\
\hline
\end{tabular}
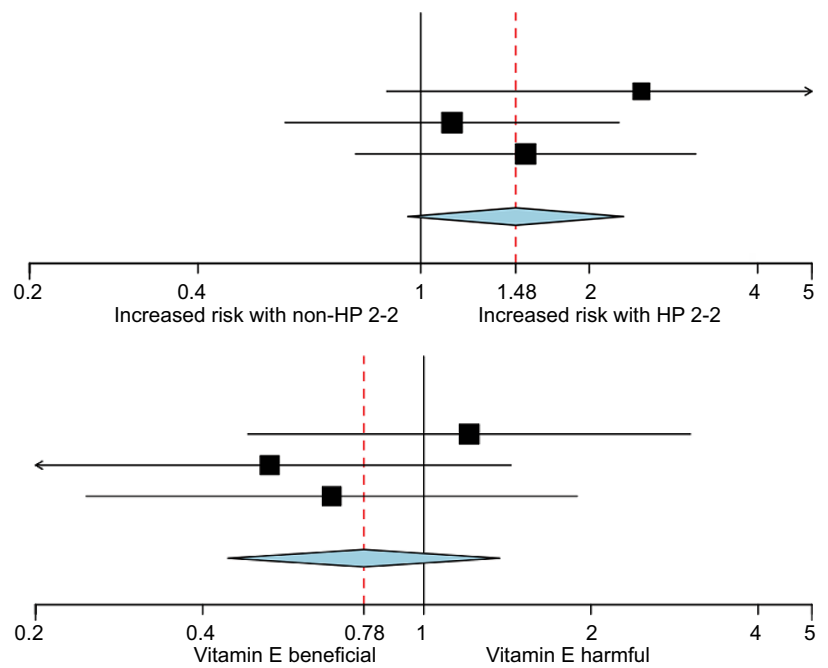

Figure $6 \mathrm{Hp}$ 2-2 genotype and risk of stroke and effect of vitamin E on stroke risk.

Notes: (A) Random effect meta-analysis of cohort and randomized controlled studies for stroke between $\mathrm{Hp}$ 2-2 and non-Hp 2-2 genotypes. (B) Random effect metaanalysis of the effects of vitamin $\mathrm{E}$ administration on stroke in diabetic patients with the $\mathrm{Hp}$ 2-2 genotype.

Abbreviations: HOPE, Heart Outcomes Prevention Evaluation; Hp, haptoglobin; ICARE, Israeli Cardiovascular Vitamin E; RCT, randomized controlled trial; WHS, Women's Health Study.

$\begin{array}{llll}\text { Studies } & \text { Estimate }(\mathbf{9 5} \% \mathbf{C l}) & \text { Ev/HP 2-2 } & \text { Ev/non-HP 2-2 } \\ & & & \\ \text { DCCT/EDIC } & & & \\ \text { EDC-ESRD } & & & \\ & 2.058(0.861-4.920) & 12 / 516 & 9 / 787 \\ \text { Overall }\left(I^{2}=0 \%, P=0.564\right) & 1.520(0.876-2.636) & 30 / 202 & 28 / 272 \\ & 1.657(1.040-2.640) & 42 / 718 & 37 / 1,059\end{array}$

Figure $7 \mathrm{Hp}$ 2-2 genotype and risk of ESRD.

Note: Random effect meta-analysis for ESRD between $\mathrm{Hp}$ 2-2 and non-Hp 2-2 genotypes.

Abbreviations: DCCT, Diabetes Control and Complications Trial; EDC, Epidemiology of Diabetes Complications; ESRD, end-stage renal disease; Hp, haptoglobin; RCT, randomized controlled trial. 
Although no significant relationship between Hp 2-2 haplotype and stroke was found in this study, previous studies have noted significant associations between the Hp genotype and stroke. One study found that the Hp 2 allele was associated with ischemic stroke in the general population. ${ }^{25}$ Furthermore, a retrospective analysis of the Helsinki Stroke Aging Memory trial of 378 patients who previously experienced a stroke showed that the combined risk of ischemic $\mathrm{CV}$ death was 2.4-fold higher among patients with at least one Hp 2 allele but only showed a nonsignificant trend toward an association with subsequent ischemic strokes. ${ }^{26}$ Interestingly, another study found that the Hp 1-1 haplotype was associated with lacunar stroke in young patients lacking silent lacunar infarcts or extensive white matter lesions. ${ }^{27}$ Likewise, a prospective study found an association between the Hp 1-1 haplotype and an increased risk of stroke in type 1 diabetic patients. ${ }^{28}$ These studies highlight that the relationship between the Hp genotype and risk of stroke might be different from coronary artery disease.

It remains to be seen whether other antioxidants may provide protection. The WAVE study showed that vitamin $\mathrm{C}$ added to vitamin $\mathrm{E}$ therapy had a detrimental effect in postmenopausal Hp 2-2 DM women as they displayed a marked decrease in luminal diameter as compared to those who received placebo. ${ }^{29}$ Preclinical studies have also demonstrated that adding vitamin $\mathrm{C}$ to vitamin $\mathrm{E}$ mitigates the protective effect of vitamin $\mathrm{E}$ on atherosclerotic plaque development. ${ }^{30,50}$ When combined with the results of other studies, this indicates that vitamin E antioxidant therapy may provide superior benefit to this patient group and that other antioxidant therapies may actually be detrimental.

This analysis is limited for certain subgroups and CVD disorders. While the trends are consistent with those seen in type 2 DM for coronary disease, only two studies have examined this relationship in type $1 \mathrm{DM}$ and/or using ESRD end point outcomes. Previously, a mechanism to explain how the Hp 2-2 genotype causes an increased risk of CV events was proposed. On the basis of preclinical and clinical data, it has been proposed that the increased risk of $\mathrm{CV}$ events associated with the Hp 2-2 genotype is due to the Hp 2 polymers causing HDL to become dysfunctional and pro-atherogenic. HDL normally functions to protect against CVD by serving as an antioxidant, modulating inflammation by preventing the production of oxidized low-density lipoprotein and facilitating reverse cholesterol transport. ${ }^{31,32}$ The serum from DM patients with the Hp 2-2 genotype has been shown to have a decreased ability to mediate reverse cholesterol transport compared to DM patients with Hp 1-1. ${ }^{13}$ It has been suggested that this is due to a synergy between Hp 2-2 and DM causing downregula- tion of the $\mathrm{Hb}$ scavenging receptor, CD163, on macrophages and monocytes, thereby resulting in the delayed clearance of the $\mathrm{Hp}-\mathrm{Hb}$ complexes in DM patients with the Hp 2-2 genotype. ${ }^{14,33}$ As Hp can bind to ApoA1, this results in an increased amount of $\mathrm{Hp}-\mathrm{Hb}$ being associated with HDL in DM patients with the Hp 2-2 genotype (Figure 8). The binding of $\mathrm{Hb}$ to HDL results in oxidative modification of HDL, a process that is mediated by the ability of iron to generate free radicals via the Fenton reaction leading to lipoprotein peroxidation. The increased iron also leads to more nitrous oxide scavenging and decomposition leading to decreased signaling. Furthermore, this causes endothelial injury and dysfunction, platelet aggregation, inflammation and increased thermogenesis, all of which lead to an increased risk of $\mathrm{CV}$ events.

The differential effect of vitamin $\mathrm{E}$ on adverse $\mathrm{CV}$ outcomes may be explained by differences in the underlying oxidative stress burden in DM patients with different Hp genotypes. Studies have shown that vitamin $E$ acts as a potent antioxidant due to its capability of scavenging free radicals and blocking oxidative reactions. ${ }^{16,17}$ In DM patients with the Hp 2-2 genotype, vitamin E administration can reverse the deleterious cascade driven by high oxidative stress in this group by balancing this increased oxidative burden thus mitigating CV risk. Low level of oxidative stress is still required to maintain normal hemostasis and metabolic function and is also crucial for facilitating various biological processes, such as its involvement in host defense against pathogens. Among patients with low oxidative stress, abolishing this protective manner of oxidation required for maintaining stability may be harmful. By disturbing the balance between pro-oxidants and antioxidants in patients with low oxidative stress, vitamin E administration may increase the risk of adverse outcomes in patients with non-Hp 2-2 genotypes who generally have low levels of oxidative stress.

The mechanisms underlying the association of the $\mathrm{Hp}$ 2-2 genotype with increased risk of renal failure are less understood. Oxidative stress has been shown to play a role in renal injury. Studies have shown that iron accumulation within lysosomes of the renal proximal tubules are increased in diabetic animal models with the Hp 2-2 genotype compared with those with other Hp genotypes, leading to poor lysosomal integrity and cell injury. ${ }^{34,51}$ Using renal quantitative T2* MRI in patients with type $1 \mathrm{DM}$, Costacou et a ${ }^{35}$ have recently shown that kidney iron deposition is significantly increased among patients with the Hp 2-2 genotype and albuminuria.

This study has several limitations. First, given the small number of available studies on this topic in the literature, the data from studies that differ in design, clinical endpoints and length of follow-up had been included. Second, the 


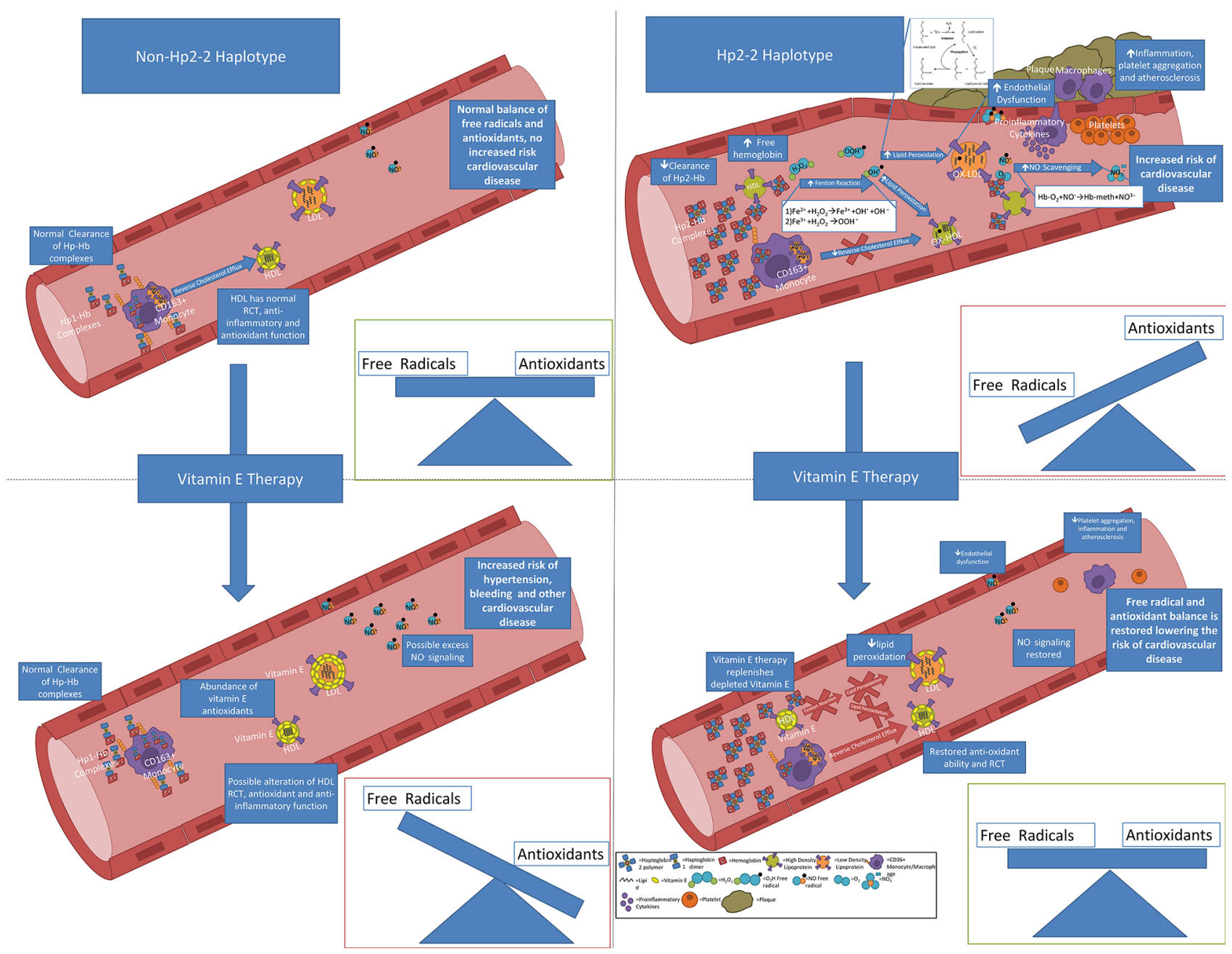

Figure 8 Mechanistic explanations of the increased risk of atherosclerosis CVD in patients with the Hp 2-2 genotype and the mechanistic rationale of using vitamin E to reduce $\mathrm{CV}$ burden in this cohort.

Notes: Top right image shows an illustration of how the $\mathrm{Hp}$ 2-2 genotype in diabetic patients leads to decreased clearance of $\mathrm{Hp}-\mathrm{Hb}$ complexes due to downregulation of the CDI63 receptor. The higher steady state of the cell-free $\mathrm{Hp}-\mathrm{Hb}$ complexes leads to the generation of ROS via the Fenton reaction subsequently to lipid peroxidation. It also leads to more binding of the $\mathrm{Hp} \mathrm{2-2-Hb}$ complexes to $\mathrm{HDL}$ and subsequent oxidation of lipoproteins. This renders $\mathrm{HDL}$ dysfunction causing increased inflammation, reduced antioxidant ability, reduced RCT, increased atherogenesis and an increased risk of CV events. The bottom right image shows how vitamin $\mathrm{E}$ therapy can reverse this cascade by maintaining the balance between antioxidant and ROS and remove the associated risk with the $\mathrm{Hp} 2-2$ genotype. The figures on the left show how vitamin E therapy can cause an increased risk of hypertension, bleeding and increased CVD in DM patients with the non- $\mathrm{Hp} 2-2$ genotype by disturbing the balance of antioxidants and ROS with administration of vitamin $\mathrm{E}$.

Abbreviations: CV, cardiovascular; CVD, cardiovascular disease; Hb, hemoglobin; HDL, high-density lipoprotein; Hp, haptoglobin; LDL, low-density lipoprotein; ROS, reactive oxygen species.

limited number of RCTs could be underpowered to show significant increase in the risk of stroke in DM patients with the Hp 2-2 genotype or to show a significant decrease in the risk of MI with vitamin $\mathrm{E}$ administration in the same population. Moreover, these RCTs did not initially aim to examine the Hp genotype, and thus the data were retrospectively analyzed. However, despite the limited number and differences between the included studies, baseline characteristics were similar, and the combined study cohorts allowed us to identify significant associations with several $\mathrm{CV}$ outcomes that are of great clinical relevance to the DM population. Finally, although attempts were made to include all journal articles matching the stated inclusion criteria, it is still possible that this analysis was subject to publication bias.

\section{Conclusion}

Globally, the number of patients with DM has increased dramatically since 1980, and the World Health Organization estimated that the prevalence has nearly doubled to $8.5 \% .{ }^{1} \mathrm{~A}$ majority of DM patients have type $2 \mathrm{DM}$ and $\sim 36 \%$ of them have the Hp 2-2 genotype. ${ }^{8,9}$ Thus, treatment targeting the increased incidence of $\mathrm{CV}$ events and increased CV mortality in DM patients with the Hp 2-2 genotype has significant potential. Administration of vitamin $\mathrm{E}$ offers a low cost means of reducing $\mathrm{CV}$ events in this population. Although it is unlikely 
that pharmaceutical companies will evaluate vitamin $\mathrm{E}$ as a potential treatment option, additional large-scale clinical trials should be pursued to evaluate its efficacy in reducing CV burden among a substantial portion of DM patients with genetically driven high oxidative stress and susceptibility to CV events.

\section{Acknowledgments}

This work was supported in part by a grant from the Israel Science Foundation to APL. The authors are solely responsible for the design and conduct of this study: all study analyses, the drafting and editing of the paper and its final contents.

\section{Disclosure}

Dr Levy is the author of a patent owned by his university that claims to predict the ability of the Hp type to predict benefit from vitamin $\mathrm{E}$. The authors report no other conflicts of interest in this work.

\section{References}

1. American Diabetes Association. Economic costs of diabetes in the U.S. in 2012. Diabetes Care. 2013;36(4):1033-1046.

2. Steinberg D, Parthasarathy S, Carew TE, Khoo JC, Witztum JL. Beyond cholesterol. Modifications of low-density lipoprotein that increase its atherogenicity. N Engl J Med. 1989;320:915-924.

3. Navab M, Anantharamaiah GM, Reddy ST, Van Lenten BJ, Ansell BJ, Fogelman AM. Mechanisms of disease: proatherogenic HDL - an evolving field. Nat Clin Pract Endocrinol Metab. 2006;2:504-511.

4. Robson EB, Polani PE, Dart SJ, Jacobs PA, Renwick JH. Probable assignment of the alpha locus of haptoglobin to chromome 16 in man. Nature. 1969;223:1163-1165.

5. Raynes JG, Eagling S, McAdam KP. Acute-phase protein synthesis in human hepatoma cells: differential regulation of serum amyloid A (SAA) and haptoglobin by interleukin-1 and interleukin-6. Clin Exp Immunol. 1991;83(3):488-491.

6. Murray RK, Connell GE, Pert JH. The role of haptoglobin in the clearance and distribution of extracorpuscular hemoglobin. Blood. 1961;17:45-53.

7. Kristiansen M, Graversen JH, Jacobsen C, et al. Identification of the haemoglobin scavenger receptor. Nature. 2001;409(6817):198-201.

8. Langlois MR, Delanghe JR. Biological and clinical significance of haptoglobin polymorphism in humans. Clin Chem. 1996;42(10):1589-1600.

9. Carter K, Worwood M. Haptoglobin: a review of the major allele frequencies worldwide and their association with diseases. Int $\mathrm{J} \mathrm{Lab} \mathrm{Hem}$. 2007;29:92-110.

10. Melamed-Frank M, Lache O, Enav BI, et al. Structure-function analysis of the antioxidant properties of haptoglobin. Blood. 2001;98(13):3693-3698.

11. Asleh R, Marsh S, Shilkrut M, et al. Genetically determined heterogeneity in hemoglobin scavenging and susceptibility to diabetic cardiovascular disease. Circ Res. 2003;92(11):1193-1200.

12. Asleh R, Guetta J, Kalet-Litman S, Miller-Lotan R, Levy AP. Haptoglobin genotype- and diabetes-dependent differences in iron-mediated oxidative stress in vitro and in vivo. Circ Res. 2005;96(4):435-441.

13. Asleh R, Miller-Lotan R, Aviram M, et al. Haptoglobin genotype is a regulator of reverse cholesterol transport in diabetes in vitro and in vivo. Circ Res. 2006;99(12):1419-1425.
14. Asleh R, Blum S, Kalet-Litman S, et al. Correction of HDL dysfunction in individuals with diabetes and the haptoglobin 2-2 genotype. Diabetes. 2008;57(10):2794-2800.

15. Asleh R, Ward J, Levy NS, Safuri S, Aronson D, Levy AP. Haptoglobin genotype-dependent differences in macrophage lysosomal oxidative injury. J Biol Chem. 2014;289(23):16313-16325.

16. Burton GW, Cheng SC, Webb A, Ingold KU. Vitamin E in young and old human red blood cells. Biochim Biophys Acta. 1986;860(1):84-90.

17. Wolf G. The discovery of the antioxidant function of vitamin E: the contribution of Henry A. Mattill. J Nutr. 2005;135:363-366.

18. Vardi M, Levy NS, Levy AP. Vitamin E in the prevention of cardiovascular disease: the importance of proper patient selection. $J$ Lipid Res. 2013;54(9):2307-2314.

19. Cahill LE, Jensen MK, Chasman DI, Hazra A, Levy AP, Rimm EB. Currently available versions of genome-wide association studies cannot be used to query the common haptoglobin copy number variant. $J \mathrm{Am}$ Coll Cardiol. 2013;62:860-861.

20. Conrad DF, Conrad DF, Pinto D, et al. Origins and functional impact of copy number variation in the human genome. Nature. 2010;464(7289): 704-712.

21. 1000 Genomes Project Consortium, Abecasis GR, Auton A, et al. An integrated map of genetic variation from 1,092 human genomes. Nature. 2012;491(7422):56-65.

22. Levy AP, Larson MG, Corey D, Lotan R, Vita JA, Benjamin EJ. Haptoglobin phenotype and prevalent coronary heart disease in the Framingham offspring cohort. Atherosclerosis. 2004;172(2):361-365.

23. Koch W, Latz W, Eichinger M, et al. Genotyping of the common haptoglobin Hp 1/2 polymorphism based on PCR. Clin Chem. 2002;48(9):1377-1382.

24. Soejima M, Koda Y. TaqMan-based real-time PCR for genotyping common polymorphisms of haptoglobin (HP1 and HP2). Clin Chem. 2008;54(11):1908-1913.

25. Ijas P, Saksi J, Soinne L, et al. Haptoglobin 2 allele associates with unstable carotid plaque and major cardiovascular events. Atherosclerosis. 2013;230(2):228-234.

26. Ijas P, Melkas S, Saksi J, et al. Haptoglobin Hp2 Variant Promotes Premature Cardiovascular Death in Stroke Survivors. Stroke. 2017;48(6): 1463-1469.

27. Staals J, Pieters BM, Knottnerus IL, et al. Haptoglobin polymorphism and lacunar stroke. Curr Neurovasc Res. 2008;5(3):153-158.

28. Costacou T, Secrest AM, Ferrell RE, Orchard TJ. Haptoglobin genotype and cerebrovascular disease incidence in type 1 diabetes. Diab Vasc Dis Res. 2014;11(5):335-342.

29. Levy AP, Friedenberg P, Lotan R, et al. The effect of vitamin therapy on the progression of coronary artery atherosclerosis varies by haptoglobin type in postmenopausal women. Diabetes Care. 2004;27(4):925-930.

30. Asleh R, Levy AP. Divergent effects of alpha-tocopherol and vitamin C on the generation of dysfunctional HDL associated with diabetes and the Hp 2-2 genotype. Antioxid Redox Signal. 2010;12(2):209-217.

31. Tall AR. An overview of reverse cholesterol transport. Eur Heart J. 1998;19(Suppl A):A31-A35.

32. Watanabe J, Chou KJ, Liao JC, et al. Differential association of hemoglobin with proinflammatory high density lipoproteins in atherogenic/ hyperlipidemic mice. A novel biomarker of atherosclerosis. J Biol Chem. 2007;282(32):23698-23707.

33. Levy AP, Purushothaman KR, Levy NS, et al. Downregulation of the hemoglobin scavenger receptor in individuals with diabetes and the $\mathrm{Hp}$ 2-2 genotype: implications for the response to intraplaque hemorrhage and plaque vulnerability. Circ Res. 2007;101(1):106-110.

34. Asleh R, Nakhoul FM, Miller-Lotan R, et al. Poor lysosomal membrane integrity in proximal tubule cells of haptoglobin 2-2 genotype mice with diabetes mellitus. Free Radic Biol Med. 2012;53(4):779-786.

35. Costacou T, Orchard TJ, Moon CH, Bae KT, Fried LF, Evans RW. Is MRI detection of kidney iron deposition increased in haptoglobin 2-2 genotype carriers with type 1 diabetes? Antioxid Redox Signal. Epub 2018 Jan 3. 
36. Blum S, Vardi M, Brown JB, et al. Vitamin E reduces cardiovascular disease in individuals with diabetes mellitus and the haptoglobin 2-2 genotype. Pharmacogenomics. 2010;11:675-684.

37. Levy AP, Hochberg I, Jablonski K,et al. Haptoglobin phenotype is an independent risk factor for cardiovascular disease in individuals with diabetes: the Strong Heart Study. J Am Coll Cardiol. 2002;40:1984-1990.

38. Roguin A, Koch W, Kastrati A, Aronson D, Schomig A, Levy AP. Haptoglobin genotype is predictive of major adverse cardiac events in the 1 -year period after percutaneous transluminal coronary angioplasty in individuals with diabetes. Diabetes Care. 2003;26:2628-2631.

39. Suleiman M, Aronson D, Asleh R,et al. Haptoglobin polymorphism predicts 30-day mortality and heart failure in patients with diabetes and acute myocardial infarction. Diabetes. 2005;54:2802-2806.

40. Milman U, Blum S, Shapira C, et al. Vitamin E supplementation reduces cardiovascular events in a subgroup of middle-aged individuals with both type 2 diabetes mellitus and the haptoglobin 2-2 genotype: a prospective double-blinded clinical trial. Arterioscler Thromb Vasc Biol. 2008;28:341-347.

41. Costacou T, Ferrell RE, Orchard TJ. Haptoglobin genotype: a determinant of cardiovascular complication risk in type 1 diabetes. Diabetes. 2008;57:1702-1706.

42. Costacou T, Ferrell RE, Ellis D, Orchard TJ. Haptoglobin genotype and renal function decline in type 1 diabetes. Diabetes. 2009;58:2904-2909.

43. Blum S, Vardi M, Levy NS, Miller-Lotan R, Levy AP. The effect of vitamin E supplementation on cardiovascular risk in diabetic individuals with different haptoglobin phenotypes. Atherosclerosis. 2010;211: $25-27$.
44. Adams JN, Cox AJ, Freedman BI, Langefeld CD, Carr JJ, Bowden DW. Genetic analysis of haptoglobin polymorphisms with cardiovascular disease and type 2 diabetes in the Diabetes Heart Study. Cardiovasc Diabetol. 2013;12:31

45. Orchard TJ, Sun W, Cleary PA, et al. Haptoglobin genotype and the rate of renal function decline in the diabetes control and complications trial/epidemiology of diabetes interventions and complications study. Diabetes. 2013;62:3218-3223.

46. Cahill LE, Levy AP, Chiuve SE, et al. Haptoglobin genotype is a consistent marker of coronary heart disease risk among individuals with elevated glycosylated hemoglobin. J Am Coll Cardiol. 2013;61:728-737.

47. Pechlaner R, Kiechl S, Willeit P, et al. Haptoglobin 2-2 genotype is not associated with cardiovascular risk in subjects with elevated glycohemoglobin-results from the Bruneck Study. JAm Heart Assoc. 2014;3:e00732.

48. Cahill LE, Jensen MK, Chiuve SE, et al. The risk of coronary heart disease associated with glycosylated hemoglobin of $6.5 \%$ or greater is pronounced in the haptoglobin 2-2 genotype. J Am Coll Cardiol. 2015;66:1791-1799.

49. Orchard TJ, Backlund JC, Costacou T, et al. Haptoglobin 2-2 genotype and the risk of coronary artery disease in the Diabetes Control and Complications Trial/Epidemiology of Diabetes Interventions and Complications study (DCCT/EDIC). J Diabetes Complications. 2016;30:1577-1584.

50. Farbstein D, Kozak-Blickstein A, Levy AP. Antioxidant vitamins and their use in preventing cardiovascular disease. Molecules. 2010;15:8098-8110.

51. Nakhoul FM, Miller-Lotan R, Awad H, et al. Pharmacogenomic effect of vitamin $\mathrm{E}$ on kidney structure and function in transgenic mice with the haptoglobin 2-2 genotype and diabetes mellitus. Am J Physiol Renal Physiol. 2009;296:F830-838.
Pharmacogenomics and Personalized Medicine

\section{Publish your work in this journal}

Pharmacogenomics and Personalized Medicine is an international, peerreviewed, open access journal characterizing the influence of genotype on pharmacology leading to the development of personalized treatment programs and individualized drug selection for improved safety, efficacy and sustainability. This journal is indexed on the American Chemical

\section{Dovepress}

Society's Chemical Abstracts Service (CAS). The manuscript management system is completely online and includes a very quick and fair peer-review system, which is all easy to use. Visit http://www.dovepress. $\mathrm{com} /$ testimonials.php to read real quotes from published authors. 